CARDIOVASCULAR MEDICINE

\title{
Left ventricular pacing improves haemodynamic variables in patients with heart failure with a normal QRS duration
}

\author{
M S Turner, R A Bleasdale, C E Mumford, M P Frenneaux, J A Morris-Thurgood
}

Heart 2004;90:502-505. doi: 10.1136/hrt.2003.011759

See end of article for authors' affiliations

Correspondence to: Professor M Frenneaux, Department of Cardiology, Wales Heart Research Institute, University of Wales College of Medicine, Heath Park Cardiff CF14 4XN, UK; scaccia@cf.ac.uk

Accepted 4 July 2003

\begin{abstract}
Objectives: To assess whether patients with congestive heart failure (CHF) and a normal QRS duration can benefit from left ventricular (VDD-LV) pacing.

Design: Cardiac resynchronisation is reserved for patients with a broad QRS duration on the premise that systolic resynchronisation is the mechanism of benefit, yet improvement from pacing correlates poorly with QRS duration. In CHF patients with a broad QRS duration, those with a high resting pulmonary capillary wedge pressure (PCWP) $>15 \mathrm{~mm}$ Hg benefit. In this acute haemodynamic VDD-LV pacing study, patients with CHF with a normal QRS duration were divided into two groups-patients with a resting PCWP $>15 \mathrm{~mm} \mathrm{Hg}$ and patients with a resting PCWP $<15 \mathrm{~mm} \mathrm{Hg}$ - to determine whether benefit is predicted by a high resting PCWP.

Patients: 20 patients with CHF, New York Heart Association functional class Ilb-IV, all with a normal QRS duration ( $\leqslant 120 \mathrm{~ms}$ ).

Interventions: Temporary pacing wires were positioned to enable VDD-LV pacing and a pulmonary artery catheter was inserted for measurement of PCWP, right atrial pressure, and cardiac output.

Results: In patients with a PCWP $>15 \mathrm{~mm} \mathrm{Hg}(\mathrm{n}=10)$, cardiac output increased from 3.9 (1.5) to 4.5 (1.65) I/min ( $<<0.01)$, despite a fall in PCWP from 24.7 (7.1) to 21.0 (6.2) $\mathrm{mm} \mathrm{Hg}(\mathrm{p}<0.001)$. In patients with a PCWP $<15 \mathrm{~mm} \mathrm{Hg}$ there was no change in PCWP or cardiac output. Combined data showed that PCWP decreased from $17.0(9.1)$ to 15.3 (7.7) $\mathrm{mm} \mathrm{Hg}$ during VDD-LV pacing $(\mathrm{p}<0.014)$ and cardiac output increased non-significantly from 4.7 (1.5) to 4.9 (1.5) ( $p=0.125)$.

Conclusions: Patients with CHF with a normal QRS duration and PCWP $>15 \mathrm{~mm} \mathrm{Hg}$ derive acute haemodynamic benefit from VDD-LV pacing.
\end{abstract}

D espite advances in drug treatment, severe congestive heart failure (CHF) still causes significant morbidity, as well as a mortality worse than that of many common cancers. $^{1-4}$ Recently, pacing for heart failure, often called cardiac resynchronisation therapy (CRT), has been shown to improve exercise capacity and quality of life and to reduce hospital readmissions. ${ }^{56}$ So far, the clinical effects of this treatment have been evaluated only among patients in whom both chronic drug refractory heart failure (New York Heart Association functional class III and IV) and a prolongation of the QRS duration to $>130 \mathrm{~ms}$ are present. Most of these patients had left bundle branch block morphology (LBBB) on ECG. The mechanism of benefit from CRT has been thought to be the restoration of systolic synchrony by using predominantly a biventricular pacing mode. However, in several acute studies left ventricular (VDD-LV) pacing has been shown to produce haemodynamic benefits that are either equal to or greater than benefits of biventricular pacing, ${ }^{7-9}$ despite increasing the QRS duration. Indeed, a reduction in QRS duration did not correlate with an improvement in haemodynamic variables. Further studies have assessed left ventricular intraventricular asynchrony and found that mechanical asynchrony exists in patients with heart failure with a normal QRS duration. ${ }^{11}$

We have previously shown in an acute pilot study that, in patients with LBBB, benefit from VDD-LV pacing was predicted by a pulmonary capillary wedge pressure (PCWP) $>15 \mathrm{~mm} \mathrm{Hg.}{ }^{12}$ In this pilot study the QRS duration did not correlate with an improvement in acute haemodynamic factors. We therefore suggested that the mechanisms by which pacing benefits such patients may include an improvement in diastolic filling.
In our current study we have assessed patients with heart failure with a normal QRS duration ( $\leqslant 120 \mathrm{~ms}$ ) who would not conventionally be considered to have significant electrical asynchrony but in other respects would be candidates for CRT. The aims of this study were to determine whether patients with a short QRS duration benefit acutely from LV pacing, and to identify whether this benefit is predicted by a PCWP $>15 \mathrm{~mm} \mathrm{Hg}$.

\section{METHODS}

The local research ethics committee approved the study. Twenty patients with heart failure in class IIb-IV with LV ejection fraction $<40 \%$ who were on stable medical treatment for heart failure and were undergoing diagnostic catheterisation were enrolled. Written informed consent was obtained from all participants. Fourteen patients had ischaemic aetiology, six had dilated cardiomyopathy, five were women, and none had more than mild mitral regurgitation. All patients except two were in New York Heart Association functional class III (one in class IIb and one in class IV). All patients except one were taking angiotensin converting enzyme inhibitors at the maximum tolerated dose (one patient was intolerant because of hypotension) and all were taking loop diuretics. Mean LV end diastolic dimension was $6.48 \mathrm{~cm}$ (range $5.3-8.6 \mathrm{~cm}$ ) and mean LV ejection fraction was $31 \%$ (range $15-40 \%$ ).

\footnotetext{
Abbreviations: $\mathrm{CHF}$, congestive heart failure; CRT, cardiac resynchronisation; $L B B B$, left bundle branch block; $L V$, left ventricular; PATH-CHF, pacing therapy in congestive heart failure; PCWP, pulmonary capillary wedge pressure
} 


\section{Acute haemodynamic study}

Acute haemodynamic studies were performed in the cardiac catheterisation laboratory with patients in the non-sedated and supine state. Temporary pacing leads were placed in the high right atrium and retrogradely through the aorta to the endocardial surface of the free wall of the left ventricle. A right ventricular (RV) pacing wire was additionally placed in a subset of patients $(n=14)$. A balloon tipped catheter was placed in the pulmonary artery to measure cardiac output by thermodilution, pulmonary capillary wedge pressure, and right atrial pressure. Baseline values were measured following catheter placement and after the patients had rested for a minimum of 10 minutes (table 1). All cardiac output data were expressed as a mean of at least three measurements, which differed by $<10 \%$. Patients were then paced in VDDLV mode with the atrioventricular delay set at the longest duration that ensured ventricular capture, with a significant change in QRS morphology. This was subsequently compared with unpaced baseline data and in a subgroup of 14 patients with VDD-RV pacing.

Intrinsic and paced PR intervals were measured from the surface ECG. The paced atrioventricular delay and the PR interval could not be directly compared, as the atrial electrode could not consistently be placed at the sinoatrial node. Hence, both were measured from the surface tracings.

\section{Statistical analysis}

Data are presented as a mean (SD) and were analysed by paired Student's $t$ test. Differences were considered significant if $\mathrm{p}<0.05$.

\section{RESULTS}

The following studies and subsequent results are presented as follows to answer the aims outlined in the introduction:

- The effects of acute temporary LV pacing for the group as a whole $(\mathrm{n}=20)$

- The effects of acute temporary LV pacing for patients with a PCWP > $15 \mathrm{~mm} \mathrm{Hg}(\mathrm{n}=10)$ compared with those with a PCWP $<15 \mathrm{~mm} \mathrm{Hg}(\mathrm{n}=10)$

- The effect of LV pacing on atrioventricular delay and QRS duration.

\section{Effects of LV pacing for the group as a whole}

There was a significant decrease in PCWP from a baseline mean (SD) of 17.0 (9.1) $\mathrm{mm} \mathrm{Hg}$ to 15.3 (7.7) mm Hg during LV pacing $(p=0.014)$ (fig 1$)$. There was no change in cardiac output (4.7 (1.5) l/min $v 4.9$ (1.5) l/min during LV pacing; $\mathrm{p}=0.13$ ).

\section{Effects of LV pacing for patients with PCWP $>15$} compared with those with PCWP $<15 \mathrm{~mm} \mathrm{Hg}$

In patients with a high baseline PCWP $>15 \mathrm{~mm} \mathrm{Hg}$ $(\mathrm{n}=10), \mathrm{LV}$ pacing produced a significant increase in cardiac output from 3.9 (1.5) l/min at baseline to 4.5 (1.7) $\mathrm{l} /$ $\min (\mathrm{p}<0.01)$, despite PCWP falling from $24.7(7.1) \mathrm{mm} \mathrm{Hg}$

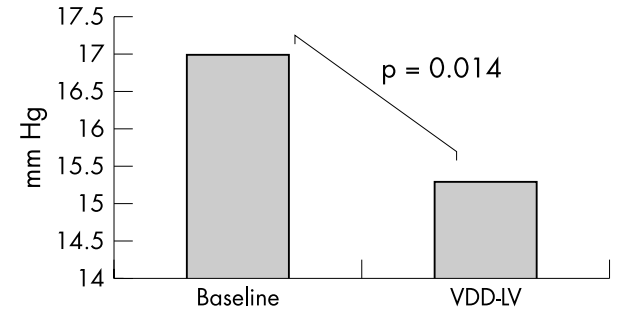

Figure 1 Graph depicting the change in pulmonary capillary wedge pressure (PCWP) for patients from both the high and low resting PCWP groups. Left ventricular (VDD-LV) pacing significantly reduced PCWP.

to $21.0(6.2) \mathrm{mm} \mathrm{Hg}(\mathrm{p}<0.001)$ (figs 2 and 3). VDD-RV pacing, at the same atrioventricular delay, was performed in 14 patients in random order, of whom seven had baseline PCWP $>15 \mathrm{~mm} \mathrm{Hg}$. VDD-RV pacing produced a significantly higher PCWP (17.6 (7.7) mm Hg v 14.7 (6.9) mm Hg; $\mathrm{p}=0.006$ ) than did VDD-LV pacing (fig 4). Cardiac output fell during VDD-RV pacing to 4.9 (1.3) l/min from 5.1 (1.5) l/ $\mathrm{min})$, although this did not reach significance $(\mathrm{p}=0.185)$.

PCWP and cardiac output did not change significantly in patients with a baseline PCWP $<15 \mathrm{~mm} \mathrm{Hg}(\mathrm{n}=10)$. The baseline PCWP remained constant with pacing (9.6 (2.4) $v$ 9.5 (3.5) $\mathrm{mm} \mathrm{Hg}$, respectively). Cardiac output was likewise unchanged (5.4 (1.1) v 5.4 (1.4) l/min) (figs 2 and 3).

\section{Effect of LV pacing on atrioventricular delay and QRS duration}

Because of the need to capture the ventricular mass we necessarily decreased the atrioventricular coupling time and delay, as shown by the fall in PR interval during pacing, in both the high and low PCWP groups (table 2). The PR intervals at baseline and during pacing were not significantly different in the high versus the low PCWP patients. As the left ventricular free wall was paced the QRS duration

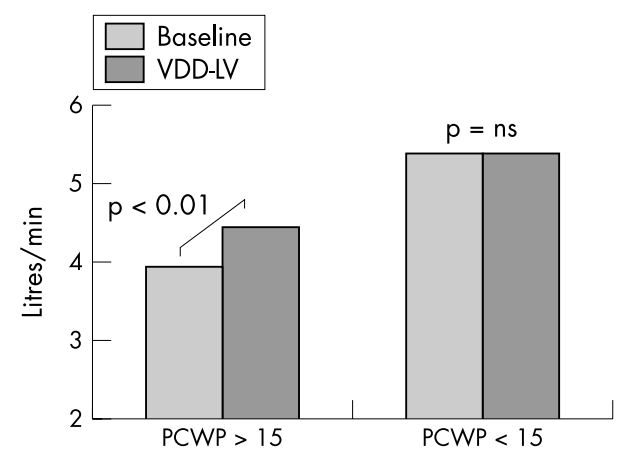

Figure 2 Change in cardiac output (CO) with VDD-LV pacing in the high and low PCWP groups. Cardiac output improved significantly in the patient group with a high resting PCWP ( $>15 \mathrm{~mm} \mathrm{Hg}$ ) but not in those with lower PCWP.

Table 1 Baseline parameters

\begin{tabular}{|c|c|c|c|c|}
\hline & \multicolumn{2}{|c|}{ High PCWP group } & \multicolumn{2}{|c|}{ Low PCWP group } \\
\hline & Mean & Range & Mean & Range \\
\hline Baseline PR (ms) & 191 & $160-240$ & 180 & $160-200$ \\
\hline Baseline QRS (ms) & 99 & $80-120$ & 96 & $70-120$ \\
\hline Resting RA pressure $(\mathrm{mm} \mathrm{Hg})$ & 12 & $7-17$ & 5 & $1-9$ \\
\hline Resting PCWP (mm Hg) & 24 & $16-35$ & 9.6 & $5-13$ \\
\hline Resting Ao sys (mm Hg) & 109 & $75-155$ & 134 & $105-200$ \\
\hline
\end{tabular}




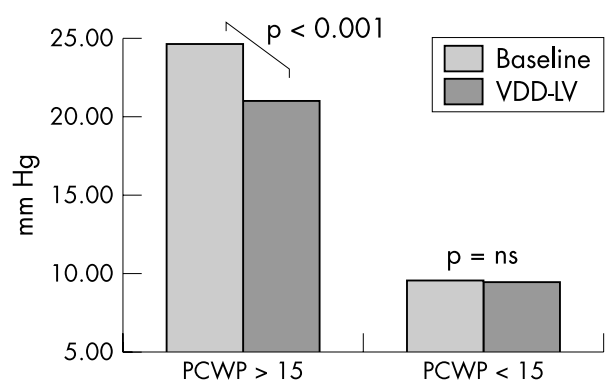

Figure 3 Change in PCWP with VDD-LV pacing in the high and low PCWP groups. VDD-LV pacing significantly reduced PCWP in the patient group with a high resting PCWP but not in those with a lower baseline PCWP.

increased relative to baseline; however, there was no significant difference between the two groups (table 2).

\section{DISCUSSION}

The important clinical message arising from this study is that patients with a normal QRS duration (that is, without LBBB) and a high resting PCWP $(>15 \mathrm{~mm} \mathrm{Hg})$ derive acute haemodynamic benefit from VDD-LV pacing. Such patients would not normally be considered for CRT. In contrast, those with a lower PCWP did not derive acute haemodynamic benefit (at least during supine rest). Patients with a higher PCWP benefited despite a major increase in the QRS duration ( $99 \mathrm{~ms}$ increasing to $156 \mathrm{~ms}$ during VDD-LV). Indeed the increase in QRS duration in this group was greater than that seen in the low PCWP group, where no benefit was seen during pacing. This provides further evidence that changes in QRS duration do not correlate well with benefits from pacing. ${ }^{73}$ The reduction in PR interval in the two groups of patients (necessary to obtain ventricular capture) was comparable.

RV pacing was detrimental in our patients in that it increased the PCWP. While this pacing mode was used primarily as a control for the reduced atrioventricular delay in this study, it may also support the implantation of resynchronisation pacemakers in patients with severe heart failure with a normal QRS duration and a conventional indication for pacing (such as atrioventricular block).

The early reports of the PATH-CHF (pacing therapy in congestive heart failure) II study, which looked at long term LV pacing, suggested that patients with a QRS duration of 120-150 ms did not derive any benefit whereas those with QRS duration $>150 \mathrm{~ms}$ did. In contrast, the one year follow up data were presented recently and also showed that patients with QRS duration between 120-150 ms and those with a QRS duration > $150 \mathrm{~ms}$ derived significant benefit from LV pacing. ${ }^{14}$ Our results suggest that this paradigm may be extended such that benefits from resynchronisation pacing in heart failure may be seen even when the QRS duration is normal.

There are a number of possible explanations for the benefits from LV pacing. These include improved mechanical synchrony; reduction in mitral regurgitation (both presystolic and systolic; and improved diastolic filling resulting from a reduction in the external constraint to the left ventricle from the pericardium and diastolic ventricular interaction ${ }^{15}$ from raised RV pressures. ${ }^{12} 16$

\section{Mechanical asynchrony}

Resynchronisation of ventricular contraction has been considered to be the principal mechanism of benefit derived from CRT. The QRS duration may be shortened when patients are paced in the biventricular mode; however,

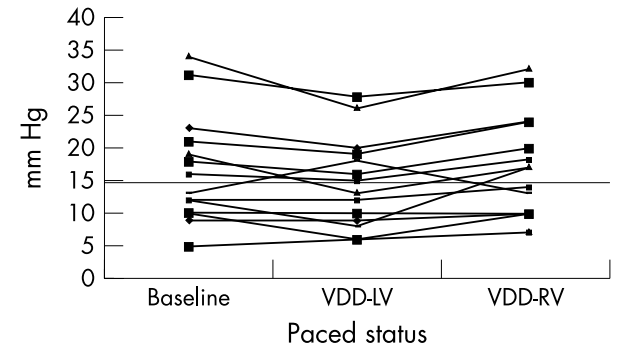

Figure 4 Change in PCWP with VDD-LV and right ventricular (VDDRV) pacing. Fourteen patients were paced in the VDD-RV mode at the same atrioventricular delay as with VDD-LV pacing. This diagram shows that (1) VDD-LV pacing reduces PCWP predominantly in patients with a high resting PCWP, and (2) VDD-RV pacing was significantly worse than VDD-LV pacing despite pacing at the same atrioventricular delay.

clinical studies have shown little or no correlation between QRS shortening during pacing and acute haemodynamic improvement..$^{72}$ In addition to asynchrony between the left and right ventricles (interventricular asynchrony), patients with CHF also have notable discoordinate LV contraction (that is, intraventricular asynchrony), which may correlate poorly with QRS duration. Recent reports have shown that biventricular pacing can improve LV intraventricular synchrony and that this predicts improvement in LV ejection fraction. ${ }^{11}$

A recent report has shown that $\mathrm{LV}$ pacing produced mechanical resynchronisation of the LV equivalent to that of biventricular pacing even though LV pacing produced no change in the myocardial activation time or "electrical synchrony", suggesting that factors other than conduction delay contribute to mechanical asynchrony. ${ }^{17}$ In addition in a study of patients with heart failure with chronically implanted pacing systems, when directly compared in the same patient, LV pacing produced greater contractility improvement than biventricular pacing for the majority of patients and improved intraventricular synchrony. ${ }^{18}$

\section{Reduction in mitral regurgitation (systolic and presystolic)}

In addition to systolic resynchronisation, it has been suggested that, by shortening the atrioventricular delay, presystolic mitral regurgitation may be reduced and that this may contribute to the haemodynamic benefit. Acute haemodynamic data have shown that atrioventricular delay shortening makes no significant contribution to the benefits of CRT. $^{8}$ Both Etienne ${ }^{19}$ and more recently Leclercq ${ }^{20}$ have provided evidence of benefits from biventricular pacing among patients in atrial fibrillation, where atrioventricular delay can definitely play no part. Furthermore, in our study VDD-LV pacing was very superior to VDD-RV pacing at the same atrioventricular delay. Systolic mitral regurgitation may be reduced by pacing but none of the patients in our study had more than mild systolic mitral regurgitation.

\section{Improved diastolic filling}

We have previously shown that in many patients with heart failure the filling of the left ventricle is impeded by an external constraint from the pericardium and by diastolic ventricular interaction from the raised RV diastolic pressure across the interventricular septum. ${ }^{21}$ Our pilot studies have shown that LV pacing reduces this external constraint and improves LV diastolic filling. ${ }^{16}$ It is uncertain at this stage as to what extent this explains the haemodynamic benefits of LV pacing.

In this study in which patients had a normal QRS duration, the acute haemodynamic benefit was strongly associated 


\begin{tabular}{|llllllll}
\hline \multicolumn{7}{l}{ Table 2} & ECG intervals \\
\hline & Base PR & QRS & RV PR & LV PR & RV QRS & LV QRS \\
\hline High PCWP group & $191(28)$ & $99(13)$ & $126(17)$ & $133(30)$ & $164(27)$ & $156(31)$ \\
Low PCWP group & $180(16)$ & $96(19)$ & $134(29)$ & $140(28)$ & $167(21)$ & $140(15)$ \\
p Value & NS & NS & NS & NS & NS & NS \\
\hline Data are mean (SD). & & & & & \\
LV, left ventricular; RV, right ventricular. & & & & & \\
\hline
\end{tabular}

with a PCWP $>15 \mathrm{~mm} \mathrm{Hg}$. This is significant, as we have previously shown that patients with heart failure with PCWP $>15 \mathrm{~mm} \mathrm{Hg}$ almost invariably have a major external constraint and diastolic ventricular interaction, whereas below $15 \mathrm{~mm} \mathrm{Hg}$ diastolic ventricular interaction is minor or absent..$^{21}$ Consistent with this, right atrial pressures were considerably higher in the high than in the low PCWP group (12.3 (3.2) v 5.1 (3.1) $\mathrm{mm} \mathrm{Hg}$, respectively; $\mathrm{p}=0.002$ ). Right atrial pressure has been shown to correlate closely with pericardial pressure and therefore provides an approximate measure of external constraint to LV filling.

\section{Clinical implications}

Our data indicate that patients with heart failure with a short QRS duration and a high PCWP gain haemodynamic benefit from VDD-LV pacing. Whether these benefits are translated into long term improvements or whether biventricular pacing offers similar improvements remains to be established. However, these patients should no longer be excluded from long term clinical end point studies of LV pacing. For such patients VDD-LV pacing may be an alternative pacing mode to biventricular pacing.

\section{ACKNOWLEDGEMENTS}

RAB, CEM, JM-T, and MPF are supported by the British Heart Foundation. MT was supported by a Bristol-Myers Squibb cardiovascular fellowship.

\section{Authors' affiliations}

M S Turner, R A Bleasdale, C E Mumford, M P Frenneaux, J A MorrisThurgood, Department of Cardiology, Wales Heart Research Institute, University of Wales College of Medicine, Heath Park, Cardiff, UK

\section{REFERENCES}

1 Anon. Effects of enalapril on mortality in severe congestive heart failure. Results of the Cooperative north Scandinavian enalapril survival study (CONSENSUS). The CONSENSUS trial study group. N Engl J Med 1987; 316:1429-35

2 Cowie MR, Mosterd A, Wood DA, et al. The epidemiology of heart failure. Eur Heart J 1997; 18:208-25.

3 Smith WM. Epidemiology of congestive heart failure. Am J Cardiol 1985;55:3A-8A.
4 Kjekshus J. Arrhythmias and mortality in congestive heart failure. Am J Cardiol 1990;65:421-81.

5 Cazeau S, Leclercq C, Lavergne T, et al. Effects of multisite biventricular pacing in patients with heart failure and intraventricular conduction delay. N Engl J Med 2001;344:873-80.

6 Abraham WT, Fisher WG, Smith AL, et al. Cardiac resynchronization in chronic heart failure. N Engl J Med 2002;346:1845-53.

7 Kass DA, Chen CH, Curry C, et al. Improved left ventricular mechanics from acute VDD pacing in patients with dilated cardiomyopathy and ventricular conduction delay. Circulation 1999;99:1567-73.

8 Auricchio A, Stellbrink C, Block M, et al. Effect of pacing chamber and atrioventricular delay on acute systolic function of paced patients with congestive heart failure. The pacing therapies for congestive heart failure study group. The Guidant congestive heart failure research group. Circulation 1999.99.2993-3001.

9 Blanc JJ, Etienne Y, Gilard M, et al. Evaluation of different ventricular pacing sites in patients with severe heart failure: results of an acute hemodynamic study. Circulation 1997;96:3273-7.

10 Kerwin WF, Botvinick EH, O'Connell JW, et al. Ventricular contraction abnormalities in dilated cardiomyopathy: effect of biventricular pacing to correct interventricular dyssynchrony. J Am Coll Cardiol 2000;35:1221-7.

11 Yu CM, Lin H, Zhang Q. High prevalence of left ventricular systolic and diastolic asynchrony in patients with congestive heart failure and normal QRS duration. Heart 2003:89:54-60.

12 Morris-Thurgood JA, Turner MS, Nightingale AK, et al. Pacing in heart failure: improved ventricular interaction in diastole rather than systolic resynchronization. Europace 2000;2:271-5.

13 Nelson GS, Curry CW, Wyman BT, et al. Predictors of systolic augmentation from left ventricular preexcitation in patients with dilated cardiomyopathy and intraventricular conduction delay. Circulation 2000;101:2703-9.

14 Butter C, Auricchio A, Stellbrink C, et al. Cardiac resynchronisation therapy stratified by QRS duration: PATH-CHF II results at one year. Pacing Clin Electrophysiol 2003;26(suppl 4II):983.

15 Morris-Thurgood JA, Frenneaux MP. Diastolic ventricular interaction and ventricular diastolic filling. Heart Fail Rev 2000;5:307-23.

16 Bleasdale RA, Turner MS, Mumford CE, et al. Left Ventricular pacing recruits preload reserve in heart failure patients: a new mechanism. J Am Coll Cardiol 2003;41(suppl A): 11110-74.

17 Leclercq C, Faris O, Tunin R, et al. Systolic improvement and mechanical resynchronization does not require electrical synchrony in the dilated failing heart with left bundle-branch block. Circulation 2002;106:1760-3.

18 Breithardt OA, Stellbrink C, Kramer AP, et al. Echocardiographic quantification of left ventricular asynchrony predicts an acute hemodynamic benefit of cardiac resynchronization therapy. J Am Coll Cardiol 2002;40:536-45.

19 Etienne Y, Mansourati J, Gilard M, et al. Evaluation of left ventricular based pacing in patients with congestive heart failure and atrial fibrillation. Am J Cardiol 1999;83:1138-40, A9.

20 Leclercq C, Walker S, Linde C, et al. Comparative effects of permanent biventricular and right-univentricular pacing in heart failure patients with chronic atrial fibrillation. Eur Heart J 2002;23:1780-7.

21 Atherton JJ, Moore TD, Lele SS, et al. Diastolic ventricular interaction in chronic heart failure. Lancet 1997;349:1720-4. 